University of New Hampshire

University of New Hampshire Scholars' Repository

Space Science Center

Institute for the Study of Earth, Oceans, and

Space (EOS)

2000

\title{
Results from the Milagrito experiment
}

\author{
A J. Smith \\ University of Maryland - College Park \\ R Atkins \\ University of Utah \\ W Benbow \\ University of California - Santa Cruz \\ D Berley \\ University of Maryland - College Park \\ M L. Chen \\ University of Maryland - College Park \\ Follow this and additional works at: https://scholars.unh.edu/ssc \\ See next page for additional authors \\ Part of the Astrophysics and Astronomy Commons
}

\section{Recommended Citation}

Results from the Milagrito experiment Smith, A. J. and Atkins, R. and Benbow, W. and Berley, D. and Chen, M. L. and Coyne, D. G. and Dingus, B. L. and Dorfan, D. E. and Ellsworth, R. W. and Evans, D. and Falcone, A. and Fleysher, L. and Fleysher, R. and Gisler, G. and Goodman, J. A. and Haines, T. J. and Hoffman, C. M. and Hugenberger, S. and Kelley, L. A. and Leonor, I. and McConnell, M. and McCullough, J. F. and McEnery, J. E. and Miller, R. S. and Mincer, A. I. and Morales, M. F. and Nemethy, P. and Ryan, J. M. and Shen, B. and Shoup, A. and Sinnis, G. and Sullivan, G. W. and Tumer, T. and Wang, K. and Wascko, M. O. and Westerhoff, S. and Williams, D. A. and Yang, T. and Yodh, G. B., AIP Conference Proceedings, 515, 441-447 (2000), DOl:http://dx.doi.org/10.1063/1.1291408

This Conference Proceeding is brought to you for free and open access by the Institute for the Study of Earth, Oceans, and Space (EOS) at University of New Hampshire Scholars' Repository. It has been accepted for inclusion in Space Science Center by an authorized administrator of University of New Hampshire Scholars' Repository. For more information, please contact Scholarly.Communication@unh.edu. 


\section{Authors}

A J. Smith, R Atkins, W Benbow, D Berley, M L. Chen, D G. Coyne, B L. Dingus, D E. Dorfan, R W. Ellsworth, D Evans, A Falcone, L Fleysher, R Fleysher, G Gisler, J A. Goodman, T J. Haines, C M. Hoffman, S

Hugenberger, L A. Kelley, I Leonor, Mark L. McConnell, J F. McCullough, J E. McEnery, R S. Miller, A I.

Mincer, M F. Morales, P Nemethy, James M. Ryan, B Shen, A Shoup, G Sinnis, G W. Sullivan, O T. Tumer, K

Wang, M O. Wascko, S Westerhoff, D A. Williams, T Yang, and G B. Yodh 


\section{AIP $\mid$ proceedings}

\section{Results from the Milagrito experiment}

A. J. Smith, R. Atkins, W. Benbow, D. Berley, M. L. Chen, D. G. Coyne, B. L. Dingus, D. E. Dorfan, R. W. Ellsworth, D. Evans, A. Falcone, L. Fleysher, R. Fleysher, G. Gisler, J. A. Goodman, T. J. Haines, C. M. Hoffman , S. Hugenberger, L. A. Kelley, I. Leonor, M. McConnell, J. F. McCullough, J. E. McEnery, R. S. Miller, A. I. Mincer, M. F. Morales, P. Nemethy, J. M. Ryan, B. Shen, A. Shoup, G. Sinnis, G. W. Sullivan, T. Tumer, K. Wang, M. O. Wascko, S. Westerhoff, D. A. Williams, T. Yang, and G. B. Yodh

Citation: AIP Conference Proceedings 515, 441 (2000); doi: 10.1063/1.1291408

View online: http://dx.doi.org/10.1063/1.1291408

View Table of Contents: http://scitation.aip.org/content/aip/proceeding/aipcp/515?ver=pdfcov Published by the AIP Publishing

\section{Articles you may be interested in}

The future of GeV-TeV y-ray astrophysics: Highlights of "Towards a Major atmospheric Cherenkov Telescope VI" workshop

AIP Conf. Proc. 516, 351 (2000); 10.1063/1.1291489

The CLUE experiment running with 8 telescopes; observations of gamma sources and runs on Moon AIP Conf. Proc. 515, 436 (2000); 10.1063/1.1291407

STACEE: Instrument performance and future plans AIP Conf. Proc. 515, 411 (2000); 10.1063/1.1291403

STACEE-32: Design, performance, and preliminary results AIP Conf. Proc. 515, 401 (2000); 10.1063/1.1291402 


\title{
Results from the Milagrito Experiment
}

\author{
A.J. Smith ${ }^{9,3}$, R. Atkins ${ }^{1}$, W. Benbow ${ }^{2}$, D. Berley ${ }^{3,10}$, \\ M.L. Chen ${ }^{3,11}$, D.G. Coyne ${ }^{2}$, B.L. Dingus ${ }^{1}$, D.E. Dorfan ${ }^{2}$,
} R.W. Ellsworth ${ }^{5}$, D. Evans ${ }^{4}$, A. Falcone ${ }^{6}$, L. Fleysher ${ }^{7}$, R. Fleysher ${ }^{7}$, G. Gisler ${ }^{8}$, J.A. Goodman ${ }^{3}$, T.J. Haines ${ }^{8}$, C.M. Hoffman ${ }^{8}$, S. Hugenberger ${ }^{4}$, L.A. Kelley ${ }^{2}$, I. Leonor ${ }^{4}$, M. McConnell ${ }^{6}$, J.F. McCullough ${ }^{2}$, J. E. McEnery ${ }^{1}$, R.S. Miller ${ }^{8,6}$, A.I. Mincer ${ }^{7}$, M.F. Morales ${ }^{2}$, P. Nemethy ${ }^{7}$, J.M. Ryan ${ }^{6}$, B. Shen ${ }^{9}$, A. Shoup ${ }^{4}$, G. Sinnis ${ }^{8}$, G.W. Sullivan ${ }^{3}$, T. Tumer ${ }^{9}$, K. Wang ${ }^{9}$, M.O. Wascko ${ }^{9}$, S. Westerhoff ${ }^{2}$, D.A. Williams ${ }^{2}$, T. Yang ${ }^{2}$, G.B. Yodh ${ }^{4}$

(The Milagro Collaboration)

(1) University of Utah, Salt Lake City, UT 84112, USA

(2) University of California, Santa Cruz, CA 95064, USA

(3) University of Maryland, College Park, MD 20742, USA

(4) University of California, Irvine, CA 92697, USA

(5) George Mason University, Fairfax, VA 22030, USA

(6) University of New Hampshire, Durham, NH 03824, USA

(7) New York University, New York, NY10003, USA

(8) Los Alamos National Laboratory, Los Alamos, NM 87545, USA

(9) University of California, Riverside, CA 92521, USA

(10) Permanent Address: National Science Foundation, Arlington, VA ,22230, USA

(11) Now at Brookhaven National Laboratory, Upton, NY11973, USA

\begin{abstract}
.
The Milagro water Cherenkov detector near Los Alamos, New Mexico is the first air shower detector capable of continuously monitoring the sky at energies between $500 \mathrm{GeV}$ and $20 \mathrm{TeV}$. Preliminary results of the Milagro experiment are presented. A predecessor of the Milagro detector, Milagrito, was operational from February 1997 to May 1998. Milagrito consisted of $2288^{\prime \prime}$ photomultiplier tubes (PMTs) arranged in a grid with a 2.8 meter spacing and submerged in 1-2 meters of water. During its operation, Milagrito collected in excess of 9 billion events with a median energy of about $3 \mathrm{TeV}$. The detector's sensitivity extends below $1 \mathrm{TeV}$ for showers from near zenith. The results of an all sky search for the Milagrito data for both transient and DC sources will be presented, including the Crab Nebula and active galaxies Markarian 501 and 421, which are known sources of $\mathrm{TeV}$ gamma-rays. Also presented will be a study of the TeV emission from gamma ray bursts (GRBs) in Milagrito's field of view detected by the BATSE experiment on the Compton Gamma-Ray Observatory.
\end{abstract}

CP515, GeV-TeV Gamma Ray Astrophysics Workshop, edited by B. L. Dingus, et al. (C) 2000 American Institute of Physics $1-56396-938-6 / 00 / \$ 17.00$ 


\section{INTRODUCTION}

The burgeoning field of Very High Energy (VHE) $\gamma$-ray astronomy has been revolutionized in the past decade by the development of imaging air Cherenkov telescopes [1-3]. These instruments detect $\gamma$-ray induced air showers by observing the Cherenkov light produced by cascading electromagnetic particles in the air. They have opened up a new window on the universe, observing $\gamma$-rays with energies greater than $1 \mathrm{TeV}$ from Active Galactic Nuclei (AGN), plerions, and supernova remnants. Despite the success of this technique, observations are limited because the instruments have a relatively narrow field and can only operate on clear moonless nights. A second type of VHE air shower detector, extensive air-shower (EAS) arrays, detect air-showers by observing the particles that reach the ground level. A conventional EAS array consists of many small particle detectors spread over a large area. Typically, only $0.5-1.0 \%$ of the total area is instrumented, resulting in very high energy thresholds of $30 \mathrm{TeV}$ or higher. Unlike ACTs, these instruments can operate continuously and observe the entire overhead sky. The Milagro collaboration has constructed an array sensitive to showers with energies below $1 \mathrm{TeV}$. The low threshold is achieved by constructing the detector at an altitude higher than most air shower detectors and using a large area water Cherenkov detector to sense particles from the shower. From February 1997 to May 1998, the Milagro collaboration operated a prototype, Milagrito.

\section{THE MILAGRITO DETECTOR}

The Milagrito detector [4] exploited a 21-million-liter man-made pond located in the Jemez Mountains near Los Alamos, New Mexico. The site is located at altitude of $2650 \mathrm{~m}$ above sea level $\left(750 \mathrm{~g} / \mathrm{cm}^{2}\right.$ atmospheric overburden $)$. The detector consisted of 228 " PMTs submerged in a covered pond. The PMTs were secured to a grid with a 2.8 meter spacing. The pond was filled approximately $\frac{1}{3}$ full with purified water covering the sensors. Data were collected at depths of $0.9,1.5$ and 2 meters above the PMTs.

Milagrito was operated with a trigger that required 100 PMTs to sense 1 or more photo-electrons (PEs) within a 300ns time window. The nominal trigger rate was $300 \mathrm{~Hz}$, but varied from $180 \mathrm{~Hz}$ to $450 \mathrm{~Hz}$ depending upon operating conditions. Snow collecting on cover increased the overburden reducing the rate, and increasing the water depth increased the rate. The bulk of the triggers, roughly $90 \%$, were due to electromagnetic showers induced by cosmic ray hadrons. About $7 \%$ of the triggers were produced by single muons with large zenith angles, and the remainder of the triggers, about $3 \%$, were unaccompanied hadron events.

Milagrito detected EAS particles via the Cherenkov light emitted by charged shower particles as they pass through the detector. Using water as a detector medium allows for the direct detection of the charged component of the showers through the Cherenkov light they produce, and also converts the abundant $\gamma$-rays 
into electrons through pair production and Compton scattering. Roughly $50 \%$ of the particles reaching the array are detected, with an average of 2 PMTs hit per particle. This efficiency for particle detection is much larger than that of sparse scintilator arrays that typically detect $<2 \%$ of the particles reaching the ground level.

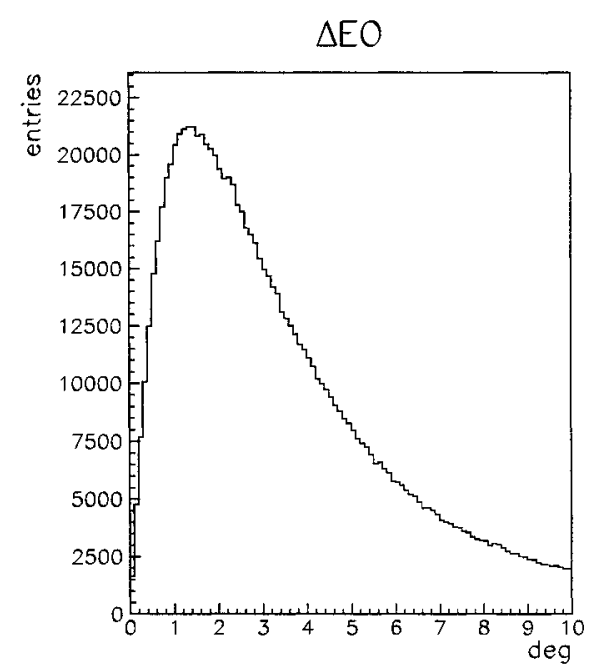

FIGURE 1. The angular resolution is estimated by dividing the array into 2 smaller arrays. Plotted is the space angle difference between the incident shower angle reconstructed by the two independent detectors. The angular resolution of the detector is approximately equal to half of $\triangle E O$.
The PMT hit times were measured to an accuracy of about 1 ns. These times are used to reconstruct the shower front of the incoming extensive air shower. The statistical error in the reconstructed shower angle is estimated by dividing the sensors grid into two overlapping sub-arrays of equal size. The space angle difference between the reconstructed directions of the even and odd arrays $(\triangle \mathrm{EO})$ is twice as large as the point spread function of the entire detector. Figure 1 is a plot of $\triangle \mathrm{EO}$ for the Milagrito detector. The distribution peaks at about $2^{\circ}$, corresponding to a detector angular resolution of about $1^{\circ}$. In practice, analyses are optimized by cutting on the number of hits include in the angle fit $\left(n_{f i t}\right)$ and choosing the binsize that maximizes the sensitivity for the background level and the spectrum of the source.

Backgrounds are estimated [5] by measuring the event distribution in local detector coordinates $(\mathrm{HA}, \delta)$ for a period of a few hours. This distribution is assumed to be constant over the entire period. The data for the period is then separated into intervals sufficiently short that the angular rotation of the earth during the interval is small compared to the detector point spread function. The background for each interval is then estimated by normalizing the (HA, $\delta$ ) distribution to the number of events collected in the much shorter interval. The process is repeated summing the signals and backgrounds for the short intervals. The estimation of the angular resolution and the background subtraction method were verified by the detection of the shadow of the Moon [6].

Milagrito was sensitive to showers produced by primary $\gamma$-rays with energies as low as $\sim 100 \mathrm{GeV}$, but the mode shower energy was about $1 \mathrm{TeV}$ for showers from zenith and typically $2-4 \mathrm{TeV}$ for showers from a source that tansits near zenith. The small size of the pond compared to the lateral extent of a typical air shower along with the poor ability of this instrument to measure the amount of energy 
deposited in the pond make the estimation of shower energy on an event by event basis nearly impossible.

\section{SEARCH FOR GAMMA-RAY POINT SOURCES}

The Crab Nebula is a well known standard candle of $\mathrm{TeV}$ gamma ray astronomy. Unfortunately, the Milagrito detector lacks sufficient sensitivity to detect this source. Detector simulations predict an excess of 1-2 $\sigma$ which is consistent with our observation of $0.8 \sigma$.

During the Milagrito data-taking period active galaxy Markarian 501 (Mrk 501) was observed by a number of groups [7-10] to be in a prolonged flare state. During this period, the flux and energy spectrum of $\gamma$-rays from Mrk 501 was well studied by the Whipple and Hegra Groups. Thus, Mrk 501 is an ideal source to use to study the sensitivity of Milagrito [11].

Figure 2 shows the significance of the observed signal in the vicinity of Mrk501. An $3.7 \sigma$ excess of events was observed at the source position. An excess of $3624 \pm 990$ events was detected for the entire observational period corresponding to an excess of $9.8 \pm 2.7 \frac{\text { events }}{\text { day }}$. The rate measured February 1997 and October 1997 can be directly compared to the measurements made by atmospheric Cherenkov telescopes. During this period, we observe a rate of $13.1 \pm 4.0 \frac{\text { events }}{\text { day }}$ which is in good agreement with the predicted $\gamma$-ray rate of $12.5 \pm 3.8 \frac{\text { events }}{\text { day }}$. We interpret this result as a detection of this well studied source and as a confirmation of our detector's performance.

A search for $\gamma$-ray point sources



FIGURE 2. Sky map for the vicinity of Mrk 501. was conducted over the northern sky between $\delta=10^{\circ}$ and $\delta=60^{\circ}$ [12]. In this search the analysis was nearly identical to the Mrk 501 analysis with, for computational reasons, square search bins instead of round. As before, the size of the bin was optimized to maximize the sensitivity of the search. The sky was divided into a $0.1^{\circ} \times 0.1^{\circ}$ grid in RA and $\delta$ defining a total of 18 million candidate source positions. Each position was searched for an excess as a signature for point source $\gamma$-ray emission. Figure 3 shows the distribution of significances for the entire sky and for a subset of 6197 independent (not overlapping) bins. In both cases, the distribution of significances has a mean consistent with 0 and a width consistent with 1 . For the 6197 non-overlapping bins, the number of total "trials" is well defined. The 
bin of highest significance has an excess corresponding to $3.8 \sigma$. The probability of detecting a signal this large in 6197 trials 0.36 . For the non-overlapping bins, the number of trials is less than the total number of entries, because neighboring bins are highly correlated. The number of effective trials for this search has not yet been calculated, but the data shows no evidence for the detection of a $\gamma$-ray point source. As simulations predict a $1.3 \sigma$ excess from the position of the Crab nebula, a $6.5 \sigma$ signal would correspond to a signal strength 5 times that of the Crab. No such signal is observed, so we conclude that no source with a Crab-like spectrum had an average flux greater than 5 times the flux of the Crab nebula during the observational epoch of Milagrito. A detailed study of the total number of effective trials taken in the search and a more stringent declination dependent upper limit will be calculated.

\section{SEARCH FOR GAMMA-RAY BURSTS}
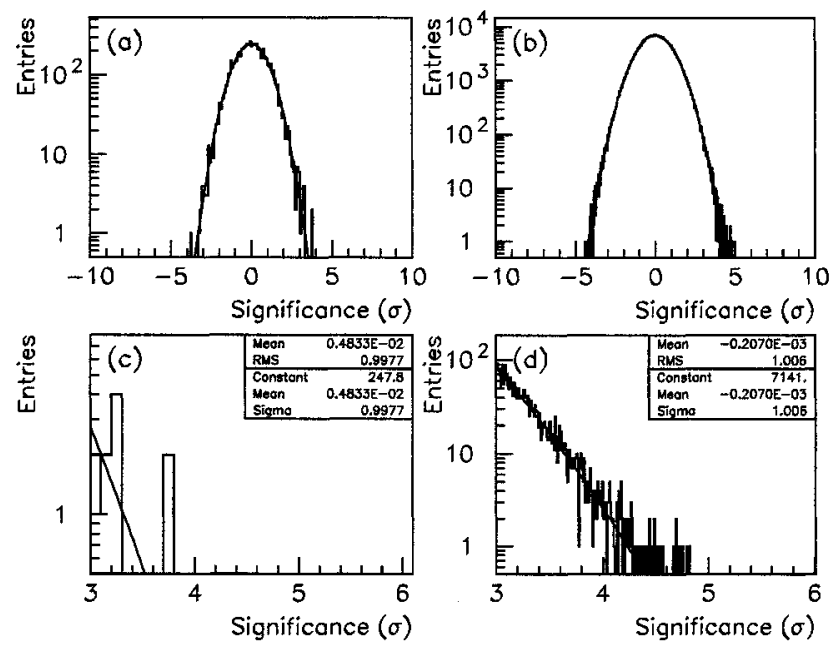

FIGURE 3. Plots (a) and (b) show the distribution of significances for 6197 independent bins and 18,000,000 overlapping bins respectively. Plots (c) and (d) show the same data as (a) and (b) with the region from $3 \sigma$ to $6 \sigma$ expanded and the fit statistics displayed
During the lifetime of Milagrito, 54 gammaray bursts (GRBs) detected by the Burst and Transient Source Experiment (BATSE) [13] were within $45^{\circ}$ of Milagrito's zenith $[15,14] . \quad$ A search was conducted for $\mathrm{TeV}$ emission coincident in time and space with the BATSE detections $[14,15]$. The search time used was T90 measured by BATSE and defined as the time over which from $5 \%$ to $95 \%$ of the detected $\gamma$-rays were detected. As the angular resolution of Milagro is much better than that of BATSE, a search was conducted for point source emission within the the BATSE $90 \%$ error radius. For this search, no $n_{f i t}$ cut was applied to the data and bins of radius $1.6 \mathrm{deg}$ were used. Unlike the search for DC sources on large backgrounds, when the backgrounds are small, larger binsizes and smaller $n_{f i t}$ cuts are optimal. The background was estimated from 2 hours of data collected before and after the 
GRB. A grid search was conducted, and the point of highest Poisson probability was taken to be the candidate GRB position. In order to establish the significance of the candidate position, an ensemble of "fake" skymaps was generated by randomly selecting events from the background distribution. These maps were searched in the same manner as the data. The probability of a background fluctuation producing a candidate of the measured excess is simply the ratio of the number to fake skymaps that contain a candidate position of equal or greater significance to the total number of fake skymaps thrown. This search procedure was fixed prior to the analysis of the data.

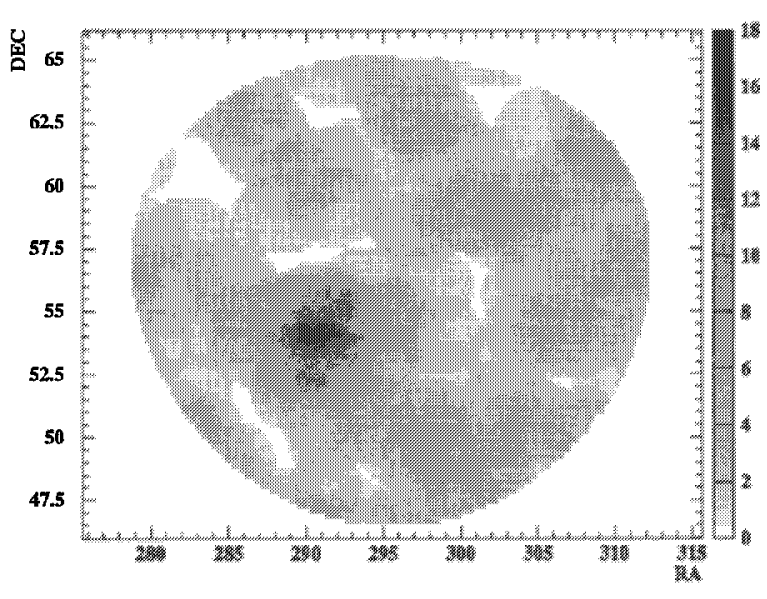

FIGURE 4. Skymap of GRB 970417a search region. The number of events within a the $1.6 \mathrm{deg}$ radius search bin is plotted for each candidate position in RA and $\delta$.
For one burst, GRB $970417 \mathrm{a}$, a substantial excess above background was detected. Figure 4 is a map of the search region GRB 970417a. The search area corrected probability for this GRB is $2.4 \times 10^{-5}$ As the search was conducted for 54 GRBs, the probability of obtaing such a low probability due to a background fluctuation in any one of the searches is $1.5 \times 10^{-3}$.

A search for 10 second duration bursts of $\gamma$-rays was conducted over the entire $\mathrm{Mi}$ -

lagrito data set [16]. The search time scale, 10 seconds was chosen to be sensitive to both GRBs and primordial black hole evaporation. The search was not confined to the BATSE defined search time and region. In this analysis, the computationally difficult search was made more efficient by only over-lapping the bins by $50 \%$ in RA, $\delta$ and time. In all, 1745105940 bins were searched. No significant excess was observed within this data set.

\section{THE MILAGRO DETECTOR}

The heart of the Milagro detector [17] is of an array of 450 PMTs deployed 1.5 meters below the pond surface and a second layer of 273 PMTs located $7 \mathrm{~m}$ below the surface. The top layer (shower layer) measures the arrival times of air-shower particles reaching the ground, while the bottom layer (muon/hadron 
layer) is used to measure the spatial distribution of the shower particles and is particularly sensitive for the detection of penetrating muons and hadrons. The shower layer is principally used to measure the direction of the incoming shower, while the muon/hadron layer will be used primarily for gamma-hadron separation. Conical "baffles" have been added to the PMTs to attenuate the late light tails that reduced the angular resolution of the prototype. Baffles also reduce the number of hits on the shower layer for single muon tracks. This will allow us to trigger the detector at a much lower threshold without being swamped by a background of single muon triggers.

In addition to the pond detector, an array of 5000 liter water tanks instrumented with a single PMT will be deployed. Because the pond is small compared with the lateral extent of typical air-showers, the additional detector array will allow for the identification of the core position dramatically improving the angular and energy resolution of the instrument.

Construction of the pond element of the Milagro detector was completed in the Fall of 1998. Data taking began in February 1999 at a reduced trigger rate to shake down the electronics and online software. Simulations indicate that Milagro will be able to measure an excess of events with significance greater than $5 \sigma$ from the Crab Nebula without gamma-hadron separation. Studies of our ability to separate $\gamma$-ray initiated showers from hadron initiated showers predict quality factors of 1.5 for the whole data set and greater than 2.5 for events with many PMTs hit [18].

\section{REFERENCES}

1. Ong, R.A. 1998, Phys. Rep., 305, 93.

2. Catanese,M., Weekes,T.C. to appear in Publications of the Astronomical Society of the Pacific.

3. Hoffman, C.M., Sinnis, C., Fleury, P., and Punch, M. 1999, to appear in Reviews of Modern Physics, 71.

4. Atkins, R., et al. 1999, submitted to Nucl. Instr. Meth. A.

5. Alexandreas, D.E., et al. 1992, Nucl. Instr. Meth. A, 311, 350.

6. Wascko M.O. et al., Proc.26 ${ }^{\text {th }}$ ICRC (Salt Lake City, 1999, OG2.1.11.

7. Samuelson, F.W., et al. 1998, Astrophys. J., 501, L17.

8. N. Hayashida et al., Ap. J. L. $\mathbf{5 0 4}$ (1998) 71.

9. Aharonian, F., et al. 1999, Astron. \& Astrophys., 342, 69.

10. Djannati-Atai, A. et al., 19th Texas Simposium Proceedings

11. Westerhoff, S. et al., Proc.26 ${ }^{\text {th }}$ ICRC (Salt Lake City, 1999, OG2.6.06.

12. Wang, K. et al., Proc.26 ${ }^{\text {th }}$ ICRC, Salt Lake City, 1999, OG2.4.31.

13. Meegan, C. A. et al, Nature,355,143 (1992).

14. McEnery, J.E. et al., This Proceedings, 1999.

15. Leonor, I. et al., Proc.26 $6^{\text {th }}$ ICRC Salt Lake City, 1999, OG2.6.06.

16. Sinnis, G. et al., Proc.26 $6^{\text {th }}$ ICRC Salt Lake City, 1999, OG2.3.07.

17. McCullough,J.F. et al., Proc.26 ${ }^{\text {th }}$ ICRC Salt Lake City, 1999, HE6.1.02.

18. Yodh G.B. et al., These Proceedings. 